\title{
Is Spreading Depolarization Characterized by an Abrupt, Massive Release of Gibbs Free Energy from the Human Brain \\ Cortex?
}

(S)AGE

\author{
Jens P. Dreier ${ }^{1,2,3}$,Thomas Isele ${ }^{4}$, Clemens Reiffurth ${ }^{1,3}$, Nikolas Offenhauser, \\ Sergei A. Kirov ${ }^{5}$, Markus A. Dahlem ${ }^{4}$, and Oscar Herreras ${ }^{6}$
}

\begin{abstract}
In the evolution of the cerebral cortex, the sophisticated organization in a steady state far away from thermodynamic equilibrium has produced the side effect of two fundamental pathological network events: ictal epileptic activity and spreading depolarization. Ictal epileptic activity describes the partial disruption, and spreading depolarization describes the near-complete disruption of the physiological double Gibbs-Donnan steady state. The occurrence of ictal epileptic activity in patients has been known for decades. Recently, unequivocal electrophysiological evidence has been found in patients that spreading depolarizations occur abundantly in stroke and brain trauma. The authors propose that the ion changes can be taken to estimate relative changes in Gibbs free energy from state to state. The calculations suggest that in transitions from the physiological state to ictal epileptic activity to spreading depolarization to death, the cortex releases Gibbs free energy in a stepwise fashion. Spreading depolarization thus appears as a twilight state close to death. Consistently, electrocorticographic recordings in the core of focal ischemia or after cardiac arrest display a smooth transition from the initial spreading depolarization component to the later ultraslow negative potential, which is assumed to reflect processes in cellular death.
\end{abstract}

\section{Keywords}

spreading depression, stroke, subarachnoid hemorrhage, intracerebral hemorrhage, traumatic brain injury, epilepsy, migraine

The Brazilian neurophysiologist Aristides Leão published a remarkable series of four articles between 1944 and 1947 (Leão 1944a, 1944b, 1947; Leão and Morison 1945). He discovered and characterized a phenomenon in the cortex of animals, the spreading depression of spontaneous activity. He found that this phenomenon was accompanied by a large slow negative voltage variation (Leão 1947) — such slow voltage variations are often referred to today as direct current (DC) shifts. He speculated that this large negative DC shift reflects pronounced depolarization of neurons, which proved correct (Canals and others 2005). Today, spreading depolarization is often used as generic term for this class of abrupt sustained nearcomplete depolarizations of neurons (Dreier 2011; Hartings and others 2011a; Oliveira-Ferreira and others 2012).

Based on his laboratory experiments, Leão coined two groundbreaking translational theories with relevance for point-of-care applications today. These theories explained the fundamental nature of two frequent and important neurological conditions: the first of his speculations is now known as the spreading depression theory of the

\footnotetext{
'Center for Stroke Research Berlin, Charité University Medicine Berlin, Berlin, Germany

${ }^{2}$ Department of Neurology, Charité University Medicine Berlin, Berlin, Germany

${ }^{3}$ Department of Experimental Neurology, Charité University Medicine Berlin, Berlin, Germany

${ }^{4}$ Institute for Theoretical Physics, Technical University Berlin, Berlin, Germany

${ }^{5}$ Department of Neurosurgery, Georgia Health Sciences University, Augusta, GA, USA

${ }^{6}$ Department of Systems Neuroscience, Cajal Institute-CSIC, Madrid, Spain
}

\section{Corresponding Author:}

Jens P. Dreier, Center for Stroke Research, Campus Charité Mitte, Charité University Medicine Berlin, Charitéplatz I, I0II7 Berlin,

Germany

Email: jens.dreier@charite.de 
migraine aura (Hadjikhani and others 2001; Lauritzen 1994; Leão and Morison 1945; Olesen and others 1981). This theory was drawn from his following conclusions:

Much has been written about vascular phenomena both in clinical epilepsy and the presumably related condition of migraine. The latter disease with the slow march of scotomata in the visual or somatic sensory sphere is suggestively similar to the experimental phenomenon here described. (Leão and Morison 1945)

The second of his translational theories linked migraine aura and stroke to spreading depolarization as their common mechanism:

The results seem to indicate that in the spreading depression of activity, a change of the same nature as one, resulting from prolonged interruption of the circulation, occurs in the cerebral cortex. The electrical sign of this change is the negative slow voltage variation. (Leão 1947)

Leão proposed a large spectrum of spreading depolarization waves characterized by a common biophysical basis and ranging from terminal events under severe ischemia to short-lasting events in the healthy well-nourished brain. His fundamental concept has recently regained attention, because there is now unequivocal electrophysiological evidence for spreading depolarizations in patients with traumatic brain injury, aneurysmal subarachnoid hemorrhage, delayed ischemic stroke after aneurysmal subarachnoid hemorrhage, malignant hemispheric stroke after middle cerebral artery occlusion, and spontaneous intracerebral hemorrhage (Dohmen and others 2008; Dreier and others 2009; Dreier and others 2006; Fabricius and others 2006; Hartings and others 2011a; Hartings and others 2011b; Nakamura and others 2010; Strong and others 2002). Moreover, the full spectrum from short- to very long-lasting negative DC shifts has been recorded in the evolution of stroke and traumatic brain injury not only in animals but also in the human brain (Dreier and others 2009; Drenckhahn and others 2012; Hartings and others 2011b; Oliveira-Ferreira and others 2010). Figure 1 shows such a progression in time and space from short- to long-lasting spreading depolarization in a human recording. Furthermore, clinical evidence from measurements of cerebral blood flow or its surrogates supports the hypothesis that spreading depolarization is the pathophysiological correlate of the migraine aura (Hadjikhani and others 2001; Lauritzen 1994; Olesen and others 1981), and a rapidly increasing body of clinical and experimental literature suggests associations between migraine with aura and stroke
(Dalkara and others 2010; Dreier 2011; EikermannHaerter and others 2012; Kurth and others 2012; OliveiraFerreira and others 2012). Last but not least, the complex relationships between epileptic activity and spreading depolarization are increasingly elucidated in the human brain (Avoli and others 1991; Dreier and others 2012; Fabricius and others 2008; Gorji and others 2001; Leão and Morison 1945; Maslarova and others 2011; OliveiraFerreira and others 2012).

In the first part of this essay, we will briefly review key features of spreading depolarizations. In the second part, we will develop the idea that the physiological state, the partial depolarization of ictal epileptic activity, spreading depolarization, and death are four fundamental Gibbs free energy states of the brain cortex. The third part will focus on the presumed electrophysiological signature of cell death, the ultraslow potential component, and its relationship with spreading depolarization. The fourth part will address clusters of repeated spreading depolarizations and their potential clinical implications.

\section{Key Features of Spreading Depolarization}

Spreading depolarization is characterized by sustained depolarization of neurons close to zero, massive ion translocation between the intracellular and extracellular compartments, extreme shunt of neuronal membrane resistance, depolarization of astroglial cells, neuronal swelling, and distortion of dendritic spines (Fig. 2) (Canals and others 2005; Dreier 2011; Risher and others 2010; Somjen 2001; Takano and others 2007). Unless spreading depolarization is preceded by arrest of spontaneous activity (Leão 1947; Oliveira-Ferreira and others 2012), it initiates spreading depression of activity by a depolarization block of action potentials (Kager and others 2002).

Spreading depolarization is observed as a large slow potential change in the extracellular space (Fig. 3) (Canals and others 2005). The duration of the slow potential change correlates with that of the intracellular depolarization of the thousands or millions of neurons in the recording area, so that it can be used as a local extracellular summary measure for the near-complete sustained depolarization of the neurons. The depolarization wave seems the more deleterious to the tissue the longer it lasts. Intracellular calcium surge, damage to mitochondria, glutamate excitotoxicity, and oxygen free radical production are among the deleterious processes that are initiated by the sustained depolarization process (Dreier 2011; Dreier and others 1998; Ohta and others 2001; Somjen 2001).

The mechanism of spread has not been elucidated. Standing theories propose different modalities of 


\section{Near-complete sustained depolarization (SD) progressing from}

\section{short- to very longlasting events in time and space in the human brain}

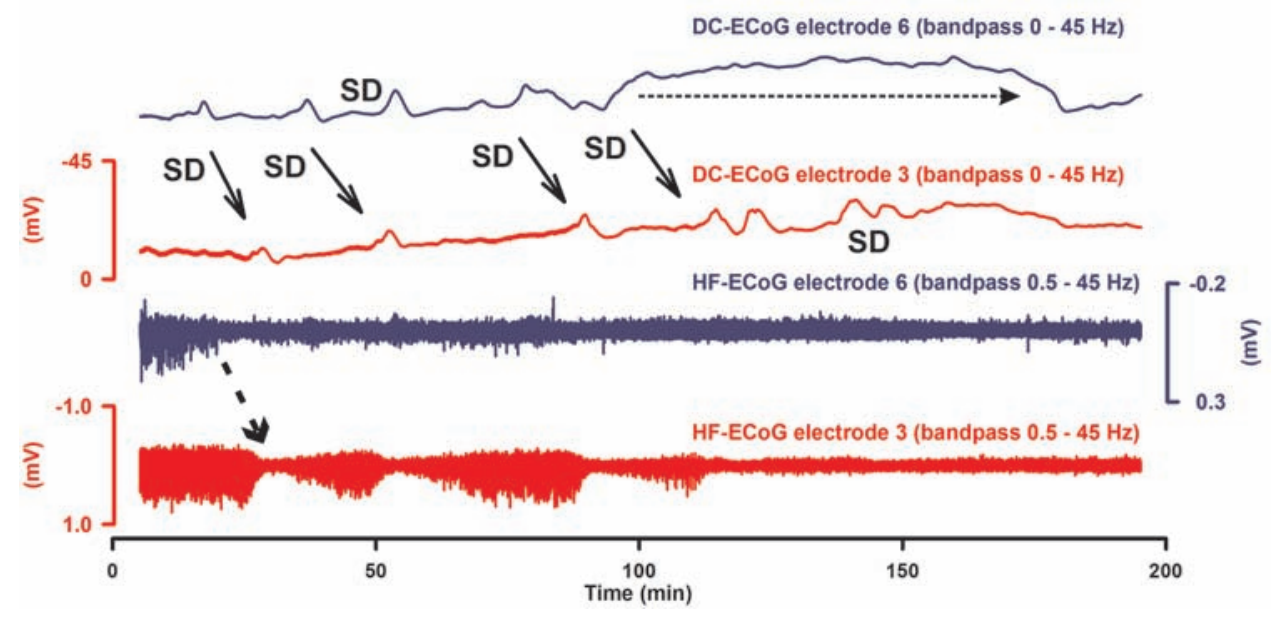

Figure I. Progression of spreading depolarizations from short-lasting to very prolonged events in the vicinity of a large intracerebral hematoma in a patient with aneurysmal subarachnoid and intracerebral hemorrhage. The upper two traces show the slow potential changes indicating the spreading depolarizations (SD) (DC-electrocorticogram [ECoG], bandpass 0-45 Hz) at electrodes 6 (blue) and 3 (red) (interelectrode distance: $3 \mathrm{~cm}$ ). Traces 3 and 4 simultaneously demonstrate the corresponding depressions of high-frequency (HF)-ECoG activity indicating depression of synaptic activity. The spreading depolarizations propagate from electrode 6 to 3 (arrows). The durations of the slow potential changes progressively increase at both electrodes with time, but this increase is more pronounced at electrode 6 (progression in time and space). The last slow potential change at electrode 6 on the right lasts for more than 60 minutes (broken arrow in trace I). In animal experiments, such a long-lasting spreading depolarization is classically termed anoxic depolarization (Bures and Buresova 1957). It should nevertheless be kept in mind that the same signal is also observed under other experimental conditions such as aglycemia or sodium pump inhibition. Note that only the first spreading depolarization is associated with spreading depression of activity propagating from electrode 6 to 3 (broken arrow between traces 3 and 4). Thereafter, the high-frequency electrocorticographic activity remains depressed at electrode 6 , a pattern that is termed persistent depression of activity. In contrast, repeated cycles of recovery and depression of activity are observed at electrode 3 for a while until persistent depression also develops at electrode 3. Neuroimaging revealed that electrode 6 was closer to a large intracerebral hemorrhage than was electrode 3. This may explain the propagation of the spreading depolarizations from electrode 6 to 3 and their longer durations at electrode 6 . Neuroimages performed before and after recording of this cluster of recurrent spreading depolarizations suggested an increase of the lesion in the time period between the scans. More details of this case are given in figure 5 of the article by Oliveira-Ferreira and colleagues (OliveiraFerreira and others 2010).

reaction/diffusion phenomena. The classic hypotheses can be grouped into one according to which the release of neuro-active substances to the extracellular space, either potassium and/or glutamate, first excites and then depresses neurons while forcing them to release more excitants (Grafstein 1956; Van Harreveld 1959). The slowness of chemical diffusion would account for the low velocity of the reaction. A later theory posited that a transcellular pathway for the reaction/diffusion via neuronal gap junctions accounts for prodromal synchronization ahead of the advancing DC and potassium front (Herreras and others 1994; Shapiro 2001). These theories are not mutually exclusive; indeed, they may actually be complementary.

\section{Biophysical Basis of Spreading Depolarization}

Based on a single neuron model, it was proposed that spreading depolarization is initiated when the neuronal cation outflux caused by ATP-dependent sodium and calcium pumps fails to compensate for the cation influx of sodium and calcium so that the net flux across the neuronal membrane persistently turns inward (Kager and others 2002). This process would lead to an abrupt change from the physiological double Gibbs-Donnan steady state toward a simple Gibbs-Donnan equilibrium (Fig. 4) (Kraig and Nicholson [1978] and chapters 3 and 19 in Somjen [2004]), which is explained in the following. 

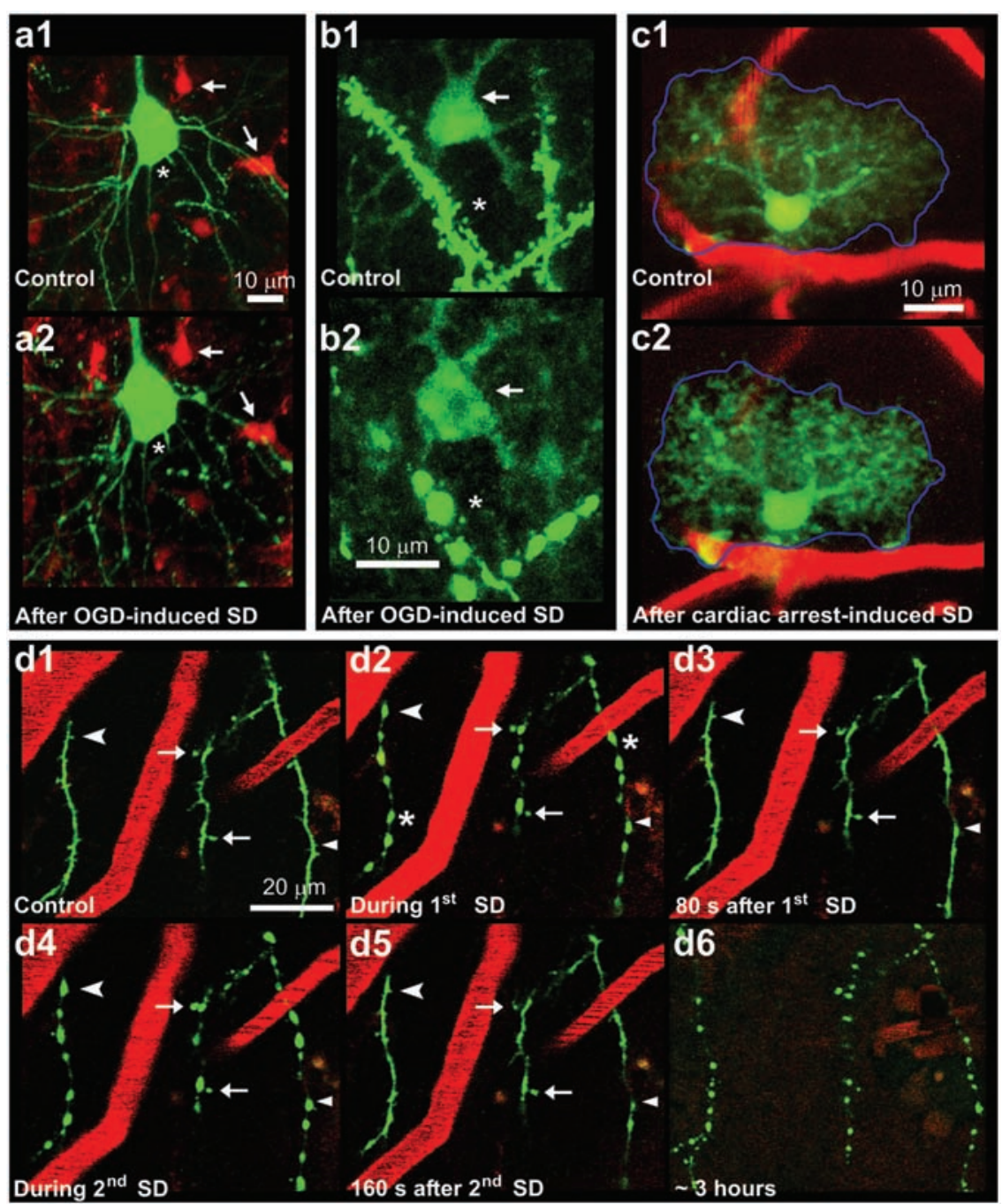

Figure 2. Astrocytes and neurons swell and dendrites bead in response to spreading depolarization (SD) under oxygen-glucose deprivation (OGD). This morphological process is termed cytotoxic edema. (al, a2) Dual photon laser scanning microscopy images of a green fluorescent protein (GFP)-expressing neuron (green) and astrocytes stained with sulforhodamine I0I (SRIOI) (red) in a mouse slice from the somatosensory cortex. The neuronal (asterisk) and astroglial somata (arrows) seen in the control image (al) become swollen and neuronal dendrites bead following spreading depolarization under oxygen-glucose deprivation (a2). (bl, b2) Dual photon images of a GFP-expressing astrocyte (arrow) and neuronal dendrites (asterisk) from the hippocampal CAI region before (bl) and 10 minutes after exposure to oxygen-glucose deprivation. Following spreading depolarization, swelling of the astroglial soma and beading of dendrites is observed (b2). The region is cropped to show the same field before and after oxygen-glucose deprivation. (cl, c2) Astrocytes swell in vivo following global ischemia-induced spreading depolarization after cardiac arrest by air embolization. Dual photon images of an astrocyte (green) from layer II/III of the somatosensory cortex and blood vessels (red) before (cl) and after ischemia-triggered spreading depolarization (c2). Despite process swelling (c2), the area of the astroglial arbor remains constant. The blue outline indicates the perimeter of the visible astroglial domain. (a-c) Modified with permission from Risher and others (2009). (dl-d6) Dendrites undergo a rapid cycle of beading and recovery coinciding with the passage of spreading depolarizations in the ischemic penumbra after local photothrombosis in mice. Low-magnification dual photon image sequence showing dendrites (green) as well as blood vessels (red; labeled with Texas Red Dextran) from layer I of the somatosensory cortex. Blood flow within vessels is indicated by streaking caused by scanning of the moving non-fluorescent red blood cells. The dendrites appear normal during control ( $\mathrm{dI}$ ) and undergo rapid beading (asterisks) and recovery coinciding with recurrent spreading depolarizations (d2-d5). Some dendritic spines are permanently lost (arrowhead), transiently lost (chevrons), or persistent (arrows) during spreading depolarization-induced dendritic beading. Appearance of dendritic beads is robust during passage of spreading depolarizations. Finally, about 3 hours after photothrombosis, the dendrites undergo terminal beading coinciding with the passage of spreading depolarization and are no longer able to recover (d6) (vessels are no longer clearly seen at this time point due to Texas Red Dextran leaking out, necessitating a reduction in the red channel gain). (d) Modified with permission from Risher and others (20I0). 


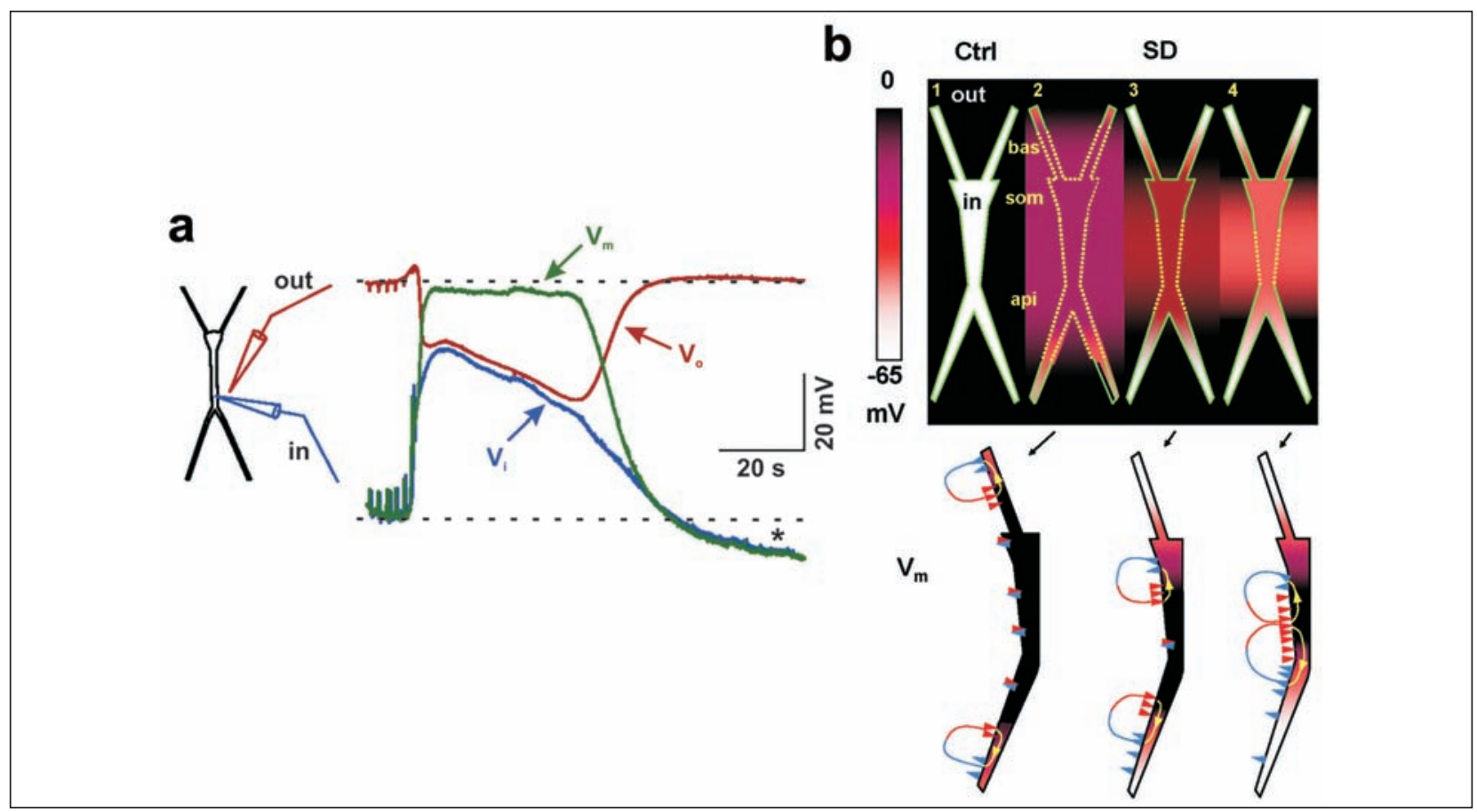

Figure 3. (a) Neurons lose their electrogenic capability during spreading depolarization due to massive depolarization. The recordings were obtained simultaneously with an intracellular electrode inside a dendrite of a pyramidal cell neuron (in blue) and outside (in red), where the negative DC signal is raised by currents from a large population of neurons. The time course of the potentials within the single cell and from the population depart from resting levels of -65 and $0 \mathrm{mV}$, respectively, then equalize on arrival of spreading depolarization and run in parallel for some 10 seconds. The transmembrane potential $(V)$ becomes near zero during the main phase of spreading depolarization. The main depolarizing phase typically lasts longer in dendrites than in their somata. At the conclusion of the spreading depolarization wave, the membrane potential of neurons becomes stronger than at rest (hyperpolarized) $(*)$, as a consequence of the work made by membrane ion pumps while regaining the potassium that was leaked out during the depolarization phase. (b) Electrogenesis of the spreading depolarization-associated DC shift in the CAI region of the hippocampus. Pyramidal cells are tightly arranged in parallel, thus optimizing the extracellular addition of currents and the spatial matching of single cell to population events with subcellular accuracy. The negative DC potential of spreading depolarization has a stereotyped depth profile marked by sharp extracellular gradients. The colored backgrounds in the upper frames represent the intracellular potential $\left(V_{j}\right)$ and the extracellular potential $(V)$, whereas the transmembrane potential $\left(V_{m}\right)$ is represented below. Intradendritic recordings provided these and the magnitude of membrane resistance decreases at each subcellular location, here represented as dashed lines in opposition to normal resistance membranes (solid lines), that is, spreading depolarization-active and spreading depolarization-spared neuron membranes, respectively. Similar colors inside and outside the neuron mean no $V_{m}$ gradient, that is, complete depolarization. Depolarization was neither complete nor homogeneous (Canals and others 2005), and it was maximal in time-varying dendritic extensions matching the low-resistance membranes and the extracellular DC shift and ion changes. Such regional depolarization in single cells establishes longitudinal gradients of depolarization and transmembrane current loops that sum in the extracellular space to build the negative DC signal (Makarova and others 20I0). When large domains of the neuron are isopotential (constant pitch in 2 and 3) axial currents (yellow lines) are only generated in the edges where internal potential gradients exist. This central cancellation of transmembrane currents in single cells is experimentally observed in currentsource density analysis of the DC profile corrected for laminar changes of resistivity. Hence, as already suggested by Leão (1947), the negative potential of spreading depolarization is created by differential polarization of neurons and specific ion conductances, very much like standard electrogenesis of spontaneous local or evoked field potentials albeit with certain peculiarities. Api, apical dendrites; bas, basal dendrites; Ctrl, control; in, intracellular space; out, extracellular space; SD, spreading depolarization; som, soma. Modified with permission from Makarova and others, Eur J Neurosci 27 (2008), published by Blackwell.

\section{The Simple Gibbs-Donnan Equilibrium}

Most cellular proteins and peptides are negatively charged because their isoelectric point is around 5 whereas the $\mathrm{pH}$ of the cytoplasm is higher. The cellular membrane is practically impermeable for these polyanions. If the cellular membrane is impermeable for polyanions inside the cell but permeable for small ions such as sodium, potassium, and chloride, a simple GibbsDonnan equilibrium will develop: the small cations potassium and sodium will be more concentrated and the 


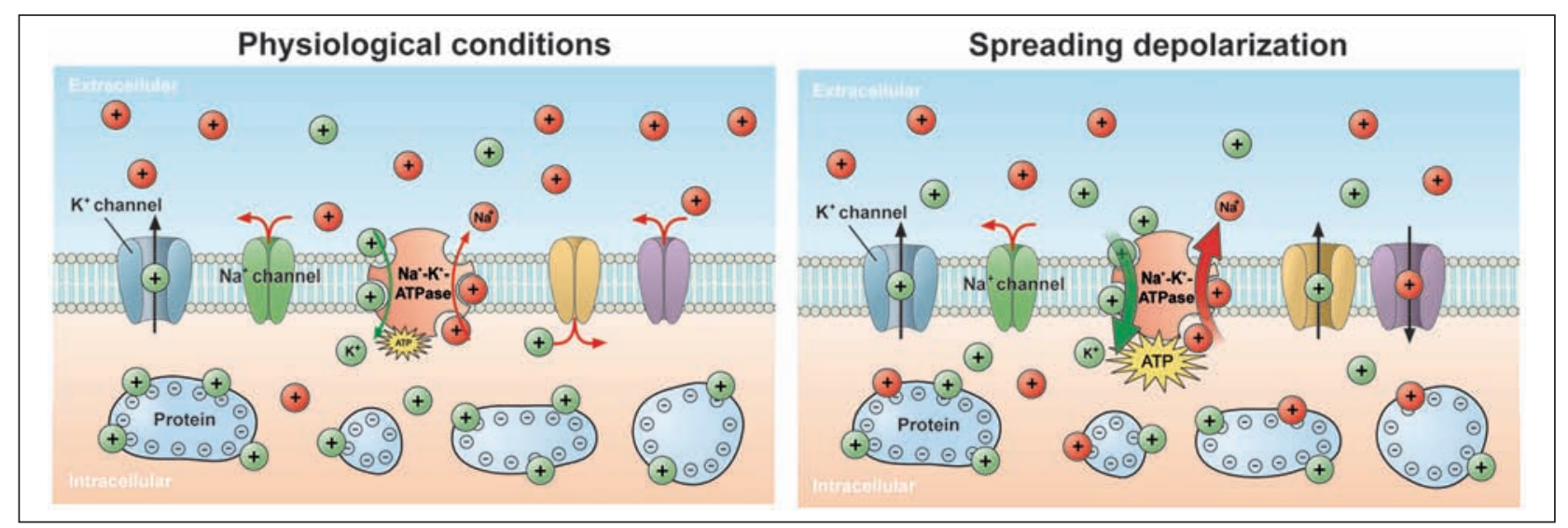

Figure 4. The concept of the physiological double Gibbs-Donnan steady state is based on a complex process of ionic immobilizations across the neuronal membrane. Sodium is actively moved by the sodium-potassium pump from the intracellular to the extracellular space against its electrochemical gradient but it can leak back down this gradient from the extra- to the intracellular space attracted by the polyanions (proteins) inside the cell. Under physiological conditions, the net effect of these two opposing processes is an accumulation of sodium in the extracellular space (also called operational immobilization of sodium in the extracellular space). Potassium moved by the sodium-potassium pump from the extra- to the intracellular space will show two fractions in the intracellular space: an operationally immobilized fraction by the pump and a fraction depending on the Gibbs-Donnan distribution (Herrera 1992). Spreading depolarization can result from two types of disturbances: (i) a persistent rise in sodium permeability leading to an increased influx of sodium into the neurons as though a greater fraction of extracellular sodium had become mobile as a consequence of a weaker capacity of the pump-leak process to exclude sodium from the intracellular space; (ii) decreased sodium pump activity resulting in a decline of the fraction of intracellular immobilized potassium by the pump, and hence a decline of the total intracellular potassium concentration (Kager and others 2002). Both a persistent increase of membrane permeability to sodium and a decline in sodium pump activity hence result in an ion distribution across the membrane that is increasingly dominated by the Gibbs-Donnan forces in contrast to the balance between Gibbs-Donnan forces and pump activity under physiological conditions. Yellow and purple pores allow for rapid sodium and calcium influx and potassium outflux during spreading depolarization. Their nature is subject to debate. In the figure, spreading depolarization occurred because of an increased sodium and calcium permeability of the membrane at the purple pore. Subsequently, the sodium-potassium pump has been activated to restore the normal ion distribution.

small anion chloride will be less concentrated inside the cell. This ionic behavior emerges because small cations are attracted to (but not bound to) the impermeant polyanions (Fig. 4). For this reason, the small cations do not cross the membrane away from the polyanions as readily as do the small anions, but remain inside. Immobilization of the polyanions thus translates into accumulation of the small cations inside the cell. In formal terms, all the small ion species are in Nernst equilibrium, that is, their concentration ratios have adjusted to fit the Nernst equation, where the potential in the Nernst equation is the Donnan potential. Of note, this Donnan potential is a transmembrane potential between the inside and outside surface of the cellular membrane while the bulk solutions on each side of the membrane are both electrically neutral, that is, both solutions have equal amounts of positive and negative ionic charges.

In the simple Gibbs-Donnan equilibrium, the product of small cations and anions inside is equal to the product of small cations and anions outside the cell, whereas the amounts of small anions and cations are unequal inside but equal outside. This implies that the sum of small anions and cations inside must be larger than the sum of small anions and cations outside. The osmotic pressure inside is thus higher than that outside. If a living cell develops a simple Gibbs-Donnan equilibrium, water will therefore enter the cell and the cell will swell. This process underlies the so called cytotoxic edema (Fig. 2). Because the cellular volume of living cells is however constant under physiological conditions, there must be a cellular process opposing the effect of the impermeant polyanions.

\section{The Sodium Pump Creates the Double Gibbs-Donnan Steady State}

For this reason, Leaf proposed the so called double Gibbs-Donnan state for various tissues where sodium is excluded from the cells as a consequence of the activity of the sodium pump. This process compensates for the attraction of the small cations by the impermeant polyanions inside the cell, creates a balance between the 
concentrations of small ions inside and outside the cell, and hence results in equal osmotic pressure inside and outside the cell (Herrera 1992; Leaf 1956). In contrast to the simple Gibbs-Donnan equilibrium, the double Gibbs-Donnan state is not at equilibrium but in a steady state because the pump constantly requires chemical energy in the form of ATP.

A number of modifications of this simple pump-leak model leads to the physiological double Gibbs-Donnan steady state of living neurons where electrogenic pumps, ion exchangers, transporters, and selective ion permeabilities maintain steady state ionic concentrations with expenditure of chemical energy (ATP) and simultaneously establish a transmembrane potential that can be rapidly changed by opening or closing specific ion channels in the membrane. Work can then be drawn from the system by the abrupt discharge and recharge of the neuronal membrane (Herrera 1992). Such electrical signals convey the information within the neurons. Of note, the flows of sodium, potassium, and calcium during such physiological electrical signaling do not lead to any changes in the ion concentration gradients across the membrane, which are detectable by the usual double-barreled ion-selective microelectrodes with a tip diameter of $3 \mu \mathrm{m}$. This is because the membrane capacitance is relatively small and only very few ions need to traverse the membrane to alter the membrane potential (Alle and others 2009). The very large majority of ions both in the cytoplasm and extracellular fluid is electrically balanced by ions with opposite polarity and does not participate in this process at the membrane. The preservation of the ion gradients under physiological conditions implies that processing information will not cause the system to lose any significant amount of Gibbs free energy in relation to the total amount stored up in the system, because the vast bulk of free electrochemical energy is contained in the ion gradients rather than in the electrical potential difference across the membrane.

This does not imply that information processing proceeds at no cost. In fact, the human brain consumes 20 to $40 \mathrm{~W}$, and at rest, information processing is the most expensive among all functions of the organism. The brain only weighs $2 \%$ of the whole body weight but consumes $20 \%$ of the whole body energy at rest (Attwell and Laughlin 2001). The sodium pump consumes about 50\% of this energy to maintain the double Gibbs-Donnan steady state during the physiological signaling (Tidow and others 2010). Nevertheless, the amount of energy being consumed at any moment in time for the signaling and maintenance of the resting potential and ion gradients is small in relation to the total electrochemical energy that is already stored up in the system in the form of physiological ion gradients. This implies that much more energy is required to reboot the system after a breakdown than to maintain the system.

\section{Life Is a Non-Equilibrium Steady State and Death a Stable Equilibrium}

It appears that brain tissue of higher animals does not survive a complete breakdown of the double GibbsDonnan steady state, which in the early stage of death could either consist of (a) a simple Gibbs-Donnan equilibrium characterized by a Donnan potential and potassium, sodium, and calcium concentrations higher inside than outside the cells or (b) no Donnan potential and equal concentrations of the small ions on both sides of the membrane when lysis of the cellular membrane allows for free diffusion of the polyanions between the media. Importantly, neither of these stable equilibria would require energy to maintain itself. Cell death incurs no costs. Whether the brain ever reaches a simple GibbsDonnan equilibrium during the early stage of cell death before cellular lysis sets in is currently unknown but this may only be of theoretical interest.

Of more practical interest are the two specific pathological states of the brain where the physiological double Gibbs-Donnan steady state is abruptly replaced by new pathological states closer to the stable equilibrium of cell death. These two pathological states are ictal epileptic activity and spreading depolarization. They are essentially characterized by an abrupt, partial loss of the ion gradients across the membrane followed by a plateau phase of variable duration during which the disturbance of ion homeostasis continues (Fig. 5) (Heinemann and Lux 1977; Kager and others 2002). Loss of the ion gradients is only moderate during ictal epileptic activity but nearcomplete during spreading depolarization (Supplementary Table 1, Fig. 5). Of note, simple suppression of spontaneous activity, due to sedatives or at the very initial stage of ischemia, for example, does not resemble an abnormal Gibbs-Donnan state, because ion gradients are essentially stable (Muller and Somjen 2000; Tanaka and others 1997).

\section{A Stepwise Decline in Gibbs Free Energy:Assumptions of the Model}

It is not trivial to calculate or measure the effective loss of Gibbs free energy in the brain when it transits from the physiological state to ictal epileptic activity to spreading depolarization to death because only the most basic features underlying these transitions are known from experiments. Nevertheless, we will attempt to calculate the relative differences between these four fundamental Gibbs free energy states of the brain based on experimental data and two simple models to illustrate the principle. Such thermodynamic states imply local minima in Gibbs free energy with states separated by an energy barrier like 


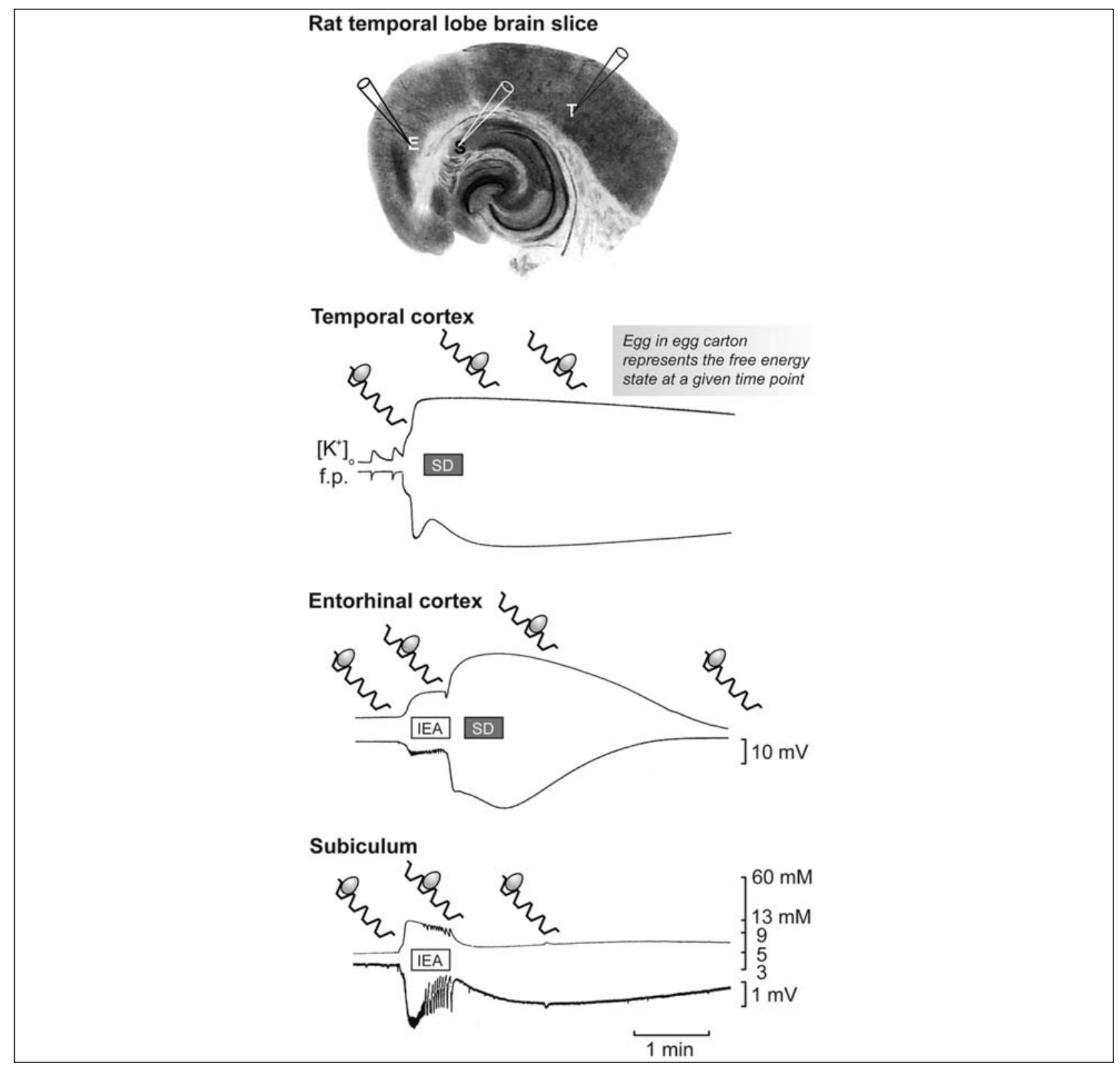

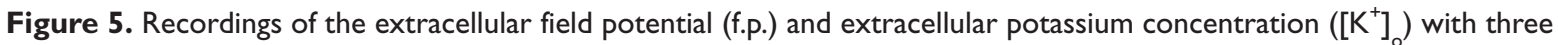
potassium-sensitive microelectrodes in the temporal neocortex $(T)$, the entorhinal cortex $(E)$, and the hippocampal subiculum (S) during ictal epileptic activity (IEA) and spreading depolarization (SD) in the presence of low-magnesium medium (Avoli and others 199I; Dreier and Heinemann 199I; Mody and others 1987). The low-magnesium medium results in overactivation of N-methylD-aspartate (NMDA) receptors because the NMDA receptor, coupled to an ionophore that is permeable to calcium, sodium, and potassium, is gated in a voltage-dependent manner by extracellular magnesium. Overactivation of NMDA receptors leads to ictal epileptic activity and spreading depolarization. In the temporal neocortex, it is seen that two interictal spikes precede the eruption of a long-lasting spreading depolarization. Shortly thereafter, ictal epileptic activity appears in the entorhinal cortex and subiculum, whereas the spreading depolarization slowly runs from the temporal neocortex to the entorhinal cortex. Both ictal epileptic activity and spreading depolarization are characterized by an abrupt, partial loss of the ion gradients across the membrane followed by a plateau phase of variable duration during which the disturbance of ion homeostasis continues. These principles are reflected here by the changes in the extracellular potassium concentration that are given at a logarithmic scale. The extracellular potassium concentration is low under physiological conditions. It increases abruptly to the ceiling level of $\sim 12 \mathrm{mM}$ during ictal epileptic activity and to $60 \mathrm{mM}$ during spreading depolarization (compare Supplementary Tables I and 2 for the other ion changes). We propose that the physiological state, ictal epileptic activity, and spreading depolarization represent local minima in Gibbs free energy with states separated by an energy barrier like eggs in an egg carton. Accordingly, a given brain region would sit in the first egg pocket during the physiological state, in the second egg pocket during ictal epileptic activity, in the third egg pocket during spreading depolarization, and in the fourth egg pocket following death. This is illustrated by the egg cartons in the figure. 
eggs in an egg carton: the eggs fall into the egg pockets and do not like sitting on the ridge between the egg pockets. In this analogy, the states are the egg pockets and the barriers are boundaries between the egg pockets. The eggs sit in either one pocket or in another (Fig. 5). Taking a thermodynamic viewpoint, the system we are describing is isothermal and isobaric. It is not isochoric because we are constructing our system from subsystems that change volume, namely, extracellular and intracellular space - the overall volume is of course conserved. In an isobaric system, the Gibbs free energy $G$, defined by $\mathrm{G}=$ $U-T S$, where $U$ is the internal energy, $T$ the (absolute) temperature, and $S$ the entropy, gives the amount of energy available to the system for performing (nonvolume-expanding) work.

In our simple models, the physiological double GibbsDonnan steady state in the cortex is termed $G_{\text {high }}$, the brain state during ictal epileptic activity $G_{\text {mid }}$, the state during spreading depolarization $G_{\text {low }}$, and that during brain death $G_{\text {bal }}$ (bal for balanced). Our aim is to calculate the contents of Gibbs free energy per volume of cortical tissue in those four different states based on the changes in cation gradients across the neuronal membrane (Supplementary Tables 1 and 2). We are aware that the total amounts of the ionic species change. Mechanisms of these changes such as uptake into subcellular compartments or astrocytes may be associated with additional energy costs. For the sake of simplicity, we have neglected these and other energy costs related, for example, to formation and cleavage of chemical bonds. We are interested only in the differences in Gibbs free energy between those states. Absolute values are of no significance to us as they are physically meaningless. For this calculation, we make several assumptions: (a) the tissue is in thermodynamic equilibrium, (b) the total amount of each ionic species is constant and the same for every state, and (c) the intracellular and extracellular solutions are ideal solutions.

The assumption that the tissue is in thermodynamic equilibrium is necessary for the Gibbs free energy to be defined. This assumption could be justified in saying that, for example, ion pumps at work, which keep the ion gradients stable in each of the states, are part of the bath, in which the actual system that we are examining is embedded. In this approximation, the effect of the ion pumps in keeping the ion concentrations constant has to be replaced by viewing the membrane as impermeable.

The second assumption is also important for our calculation. The Gibbs free energy of a solution is given by two terms: (a) the enthalpy of solution, which is given by the energy necessary to break free atoms or ions from their crystal lattice and attract the solvent molecules that surround a solute in the solution and (b) the entropy of mixing (times the absolute temperature). As we assume that the total amount of each ionic species is constant, their change from state to state will take place via ions crossing the membrane. When considering only the differences in Gibbs free energy, we can neglect the first term (enthalpy of solution) completely, because when the overall amount of each ionic species is assumed constant, so too is the enthalpy of solution. Hence, it drops out, when taking the difference. This implies that we only need to calculate the entropy of mixing for each state.

The third assumption is also important to make the Gibbs free energy accessible to a simple calculation. In assuming an ideal solution, we assume that volume and enthalpy of the solution is equal to the sum of volume and enthalpy of its constituents, so that there is no additional term due to volume and enthalpy changes when the concentration of an ionic species changes.

Overall, our assumptions say that all contributions to the Gibbs free energy in this setting come about by having the different ionic species in unequal concentrations to both sides of the membrane. The differences in Gibbs free energy are generated by the deviation of these distributions from the balanced-out distribution, as it costs energy to transfer ions against a chemical gradient.

An additional term is given by the energy that is stored in the voltage across the membrane. There is a slight inconsistency here. The membrane should be permeable for a membrane voltage to exist, but we have considered it impermeable to calculate two separate entropies of solution, one for the intracellular component and one for the extracellular compartment. In this sense, we regard the voltage as an additional feature of the state and neglect the mechanism of its generation. To estimate the amount of energy per volume stored in the electric field across the membrane, one needs to know the total membrane surface per volume, which is calculated.

The values for the concentrations and the fraction of extracellular space in a certain state are taken from experimental data (Supplemental Table 2). The only exceptions are the data for the state where the concentrations are completely balanced out (death $/ G_{\text {bal }}$ ). The concentrations for this state are calculated by taking the concentrations for $G_{\text {low }}$ and equally distributing the ions to all of intracellular and extracellular space assuming partial cellular lysis with free diffusion of polyanions across the membrane.

We assume two different-very simplified - geometries of our model neuropil, the first being that the neuronal dendrites are cylindrical and arranged as in Figure 6a, the second being that the neuronal dendrites are cuboid with a quadratic base as in Figure 6d. The calculation of the geometries, based on Figure $6 \mathrm{~b}$ and e, and the calculation of the Gibbs free energies, based on the ion concentration changes given in Supplementary Table 2, are performed in the Supplemental Material. Moreover, the contribution of the energy stored in the electric field is calculated there. 
a

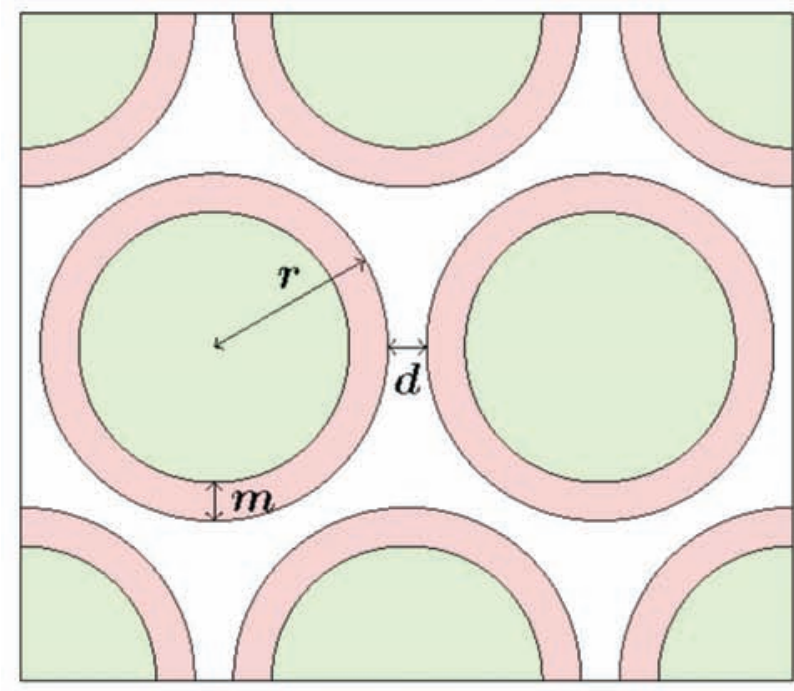

C

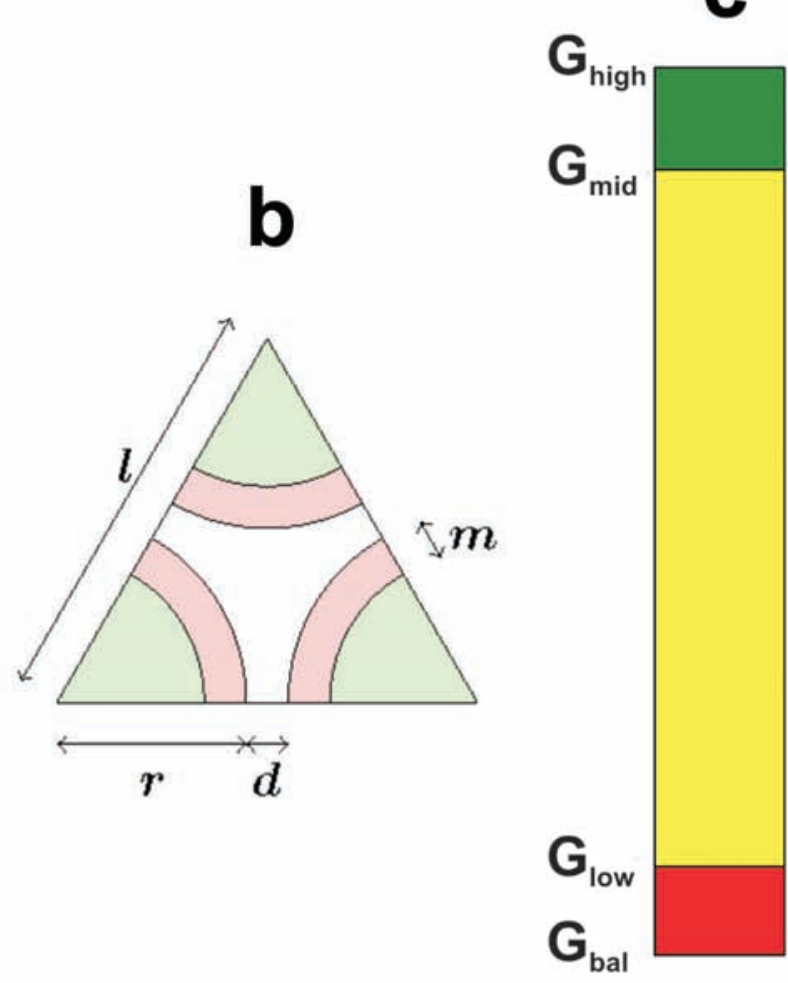

d
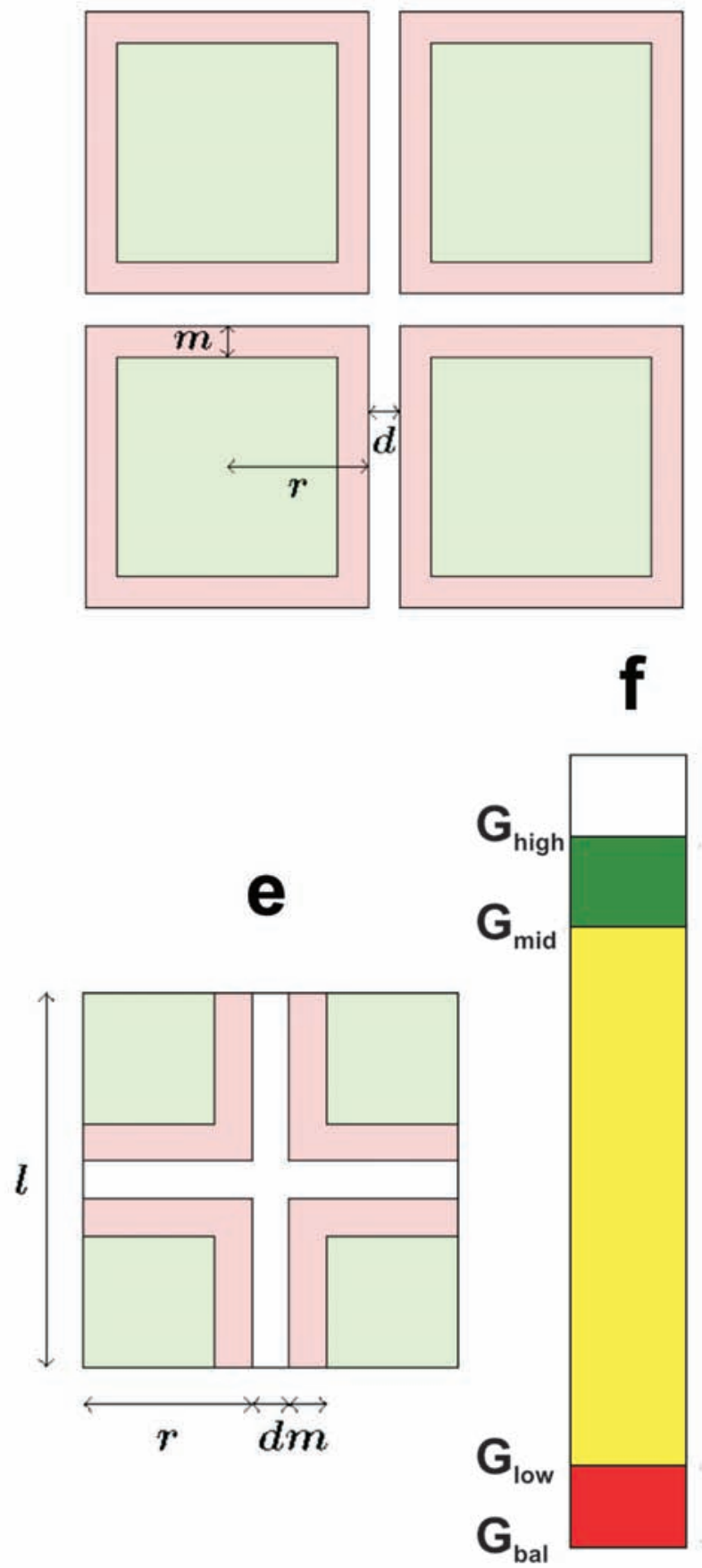

Figure 6. (a to $f$ The complex geometry of the neuropil is approximated by two simple models, the cylindrical model and the cuboid model, to calculate the changes in Gibbs free energy between the physiological state $\left(G_{\text {high }}\right)$, ictal epileptic activity $\left(G_{\text {mid }}\right)$, spreading depolarization $\left(G_{\text {low }}\right)$, and death $\left(G_{\text {bal }}\right)$. The first row in (a) and (d) shows the hypothetical arrangement of the cells. (b and e) Below, the respective unit cells are illustrated. d, distance between the cells (Van Harreveld and others 1965); I, length of the unit cell; $\mathrm{m}$, membrane diameter; $r$, radius of the unit cell. The geometrical parameters are further explained in the text. The columns (c) and (f) provide a visualization of the calculated values. Note that there is only a marginal dependence on the type of geometrical approximation. 
Together with the contribution of the changes in the electric field, we find the following differences in total Gibbs free energy between the different states for the cylindrical arrangement (Fig. 6c):

$$
\begin{gathered}
G_{\text {high }}-G_{\text {mid }}=2.8 \mathrm{~J} / l, \\
G_{\text {mid }}-G_{\text {low }}=19.2 \mathrm{~J} / l, \\
G_{\text {low }}-G_{\text {bal }}=2.4 \mathrm{~J} / l,
\end{gathered}
$$

whereas the total differences for the cuboid arrangement (Fig. 6f) is

$$
\begin{aligned}
& G_{\text {high }}-G_{\text {mid }}=2.8 \mathrm{~J} / \mathrm{l}, \\
& G_{\text {mid }}-G_{\text {low }}=16.6 \mathrm{~J} / \mathrm{l}, \\
& G_{\text {low }}-G_{\text {bal }}=2.5 \mathrm{~J} / \mathrm{l} .
\end{aligned}
$$

Note that the dependence of the overall differences on the chosen cell arrangement is negligible in qualitative terms. For the calculation it is necessary to choose a simplified cell arrangement, but in the end - at least for the two arrangements chosen here - the result does not depend much on the particular arrangement, so that one could argue that these results might hold to good approximation for more complex arrangements found in real neuropil.

As expected, the Gibbs free energy shrinks the more the ions are equally distributed. This is a generic feature of the negative mixing entropy, the reason being that the entropy is highest (and thus the Gibbs free energy lowest) when the disorder in the system is the largest. This disorder occurs when each ionic species has equal concentrations on both sides of the membrane. Here, the calculation has been expanded insofar as the volume fractions of both compartments are also subject to change. The calculations in the Supplemental Material also reveal that the changes in the electrical field account for less than $2 \%$ of the amount of Gibbs free energy lost in the transition from $G_{\text {high }}$ to $G_{\text {low }}$ in both geometrical arrangements.

\section{Experimental Verification of the Theoretical Concept}

Our models illustrate the principle of how the physiological brain state can be interpreted as a state of high Gibbs free energy $\left(G_{\text {high }}\right)$ whereas ictal epileptic activity $\left(G_{\text {mid }}\right)$ and spreading depolarization $\left(G_{\text {low }}\right)$ are states of the living brain cortex characterized by reduced Gibbs free energy with spreading depolarization close to the death $\left(G_{\mathrm{bal}}\right)$ of the system. In this way, spreading depolarization $/ G_{\text {low }}$ may be interpreted as a twilight state of the cortex during which it is decided whether the neurons will survive or die. Full recovery from this twilight state is possible up to a certain point in time, termed the commitment point (Dreier [2011] and chapter 19 in Somjen [2004]). Recovery from spreading depolarization $/ G_{\text {low }}$ largely depends on proper function of the ATP-dependent sodium pump (LaManna and Rosenthal 1975). During spreading depolarization $/ G_{\text {low, }}$, the energy demand of the pump increases so markedly that the tissue ATP concentration falls to about $50 \%$ of the normal level, although in healthy tissue the supply with oxidative substrates increases by the depolarization-induced increase in blood flow (Lauritzen 1994; Mies and Paschen 1984; Selman and others 2004). If the sodium pump fails, spreading depolarization $/ G_{\text {low }}$ will become terminal, and neurons will die $\left(G_{\text {bal }}\right)$. The sodium pump can fail if there is insufficient ATP production, as in hypoxia, hypoglycemia, or ischemia, or it can fail if the pump is pharmacologically inhibited by ouabain for example.

The difference in Gibbs free energy that we estimated can be experimentally tested in brain slices or the isolated retina. Both preparations are devoid of a blood circulation, so the blood circulation cannot interfere. The Gibbs free energy lost in the transition from $G_{\text {high }}$ to $G_{\text {low }}$ is converted to heat. This should result in a spreading wave front of heat radiation in the tissue during spreading depolarization. If we assume the specific heat capacity per volume of tissue to be approximately that of water

$$
c_{p} / v=4.183 \frac{\mathrm{kJ}}{\mathrm{kg} \cdot \mathrm{K}} / 1.006 \frac{1}{\mathrm{~kg}}=4.158 \frac{1}{\mathrm{~kg}}
$$

the temperature change should amount to

$$
\Delta T \approx 20 \frac{\mathrm{J}}{1} / 4.158 \frac{\mathrm{kJ}}{1 \cdot \mathrm{K}}=4.8 \mathrm{mK} .
$$

Temperature measurements during spreading depolarization were performed by Tasaki and Byrne in the isolated retinae of the bullfrog and the toad using a very sensitive thermal detector (Tasaki and Byrne 1991). In these experiments, spreading depolarization was validated electroretinographically (Martins-Ferreira and others 1974). Two heat sensors were located between the stimulating and recording electrodes. A spreading depolarization-induced rise in temperature at the sensors invariably preceded the onset of the DC shift at the recording electrode and moved between the two sensors at a rate of $\sim 4 \mathrm{~mm} / \mathrm{min}$. This rise in temperature lasted at a fixed point for 1 to 2 minutes. Quantitatively, the authors found that the maximum value in the rate of temperature ranged between 5 and 15 $\mathrm{mK} / \mathrm{min}$ during the phase of enhanced heat production. 
Unfortunately, these are the only exact numbers mentioned in the article for temperatures measured. Putting these values together, however, we obtain a total rise in temperature of between 5 and $30 \mathrm{mK}$ during spreading depolarization. This temperature rise and even the order of its magnitude correspond closely with the value we calculated.

That the values measured are somewhat larger than those we calculated is not surprising, as our theoretical model did not include a number of processes such as the anion concentration changes. The chloride gradient across the neuronal membrane undergoes a near-complete breakdown in a fashion similar to that of the cation gradients, but the exact magnitude of the chloride changes is more controversial (Hansen and Zeuthen 1981; Kraig and Nicholson 1978; Windmuller and others 2005). Moreover, heat will be produced shortly after the abrupt onset of spreading depolarization when neurons and astrocytes start reestablishing the ion gradients and re-uptaking the released transmitters such as glutamate. Additional recruitment of ion pumps and transporters will consume energy and will further increase heat radiation of the tissue because the efficiency of these processes is limited. Enhanced versions of the experiment by Tasaki and Byrne might therefore help verify the conclusions of our model. For example, energy metabolism could be blocked by inhibitors selectively targeting mitochondrial ATP production or respiratory complexes. The heat production associated with the massive ion translocation could then be isolated from that associated with the activated energy metabolism. Inhibitors of energy metabolism potently induce spreading depolarization, and this could be exploited in such experiments (Gerich and others 2006). In a similar fashion, the contribution of pumps or transporters to the heat radiation could be isolated by using selective inhibitors.

\section{Ultraslow Potential Components}

The transition from the twilight state spreading depolarization $/ G_{\text {low }}$ to cell death $/ G_{\text {bal }}$ implies that the typical, abruptly developing terminal spreading depolarization in the cortex or the basal ganglia (Umegaki and others 2005) is composed of two negative slow voltage components. The initial component reflects spreading depolarization $/ G_{\text {low }}$ whereas the later component is an index of the cell death $/ G_{\text {bal }}$. This later component is termed the ultraslow voltage component.

In focal ischemia in the cortex, it is particularly interesting to study the relationship between those two voltage components where the first spreading depolarization wave runs from the core of the malperfused tissue to the ischemic penumbra to the normally perfused, surrounding cortex against the gradients of oxygen, glucose, and perfusion. This first spreading depolarization starts 2 to 5 minutes after the focal arrest of the circulation (Astrup and others 1977; Leão 1947) and initiates the countdown to cell death (Dreier 2011). Further spreading depolarizations originate thereafter at the rim of the permanently depolarized core to invade the penumbra and recruit further tissue into the necrotic core (Branston and others 1977; Dijkhuizen and others 1999; Hossmann 1994; Mies and others 1993; Nedergaard and Astrup 1986; Shin and others 2006). Circling of such spreading depolarizations around the ischemic core may be involved in the progressive growth of infarction (Nakamura and others 2010). Notably, there are quantitative but no qualitative differences between the mechanisms of the first and the subsequent spreading depolarizations in focal ischemia (Dijkhuizen and others 1999; Dreier 2011; Mies and others 1993).

Cell death $/ G_{\text {bal }}$ is faster and more pronounced in the ischemic core than in the penumbra as reflected in histological sections by a larger ratio of necrotic to apoptotic cell death in the core region than in the penumbra (Charriaut-Marlangue and others 1996). Consistent with this notion, the ultraslow negative voltage shows even larger amplitude than the initial spreading depolarization component in the ischemic core, but its amplitude decreases toward the penumbra (Fig. 7a). In the penumbra, this produces the characteristic picture of recurrent, prolonged spreading depolarizations riding on a shallow negative ultraslow potential. This potential is presumably generated by local factors related to increasing cell death in the penumbra and is not simply the result of volume conduction from the remote core, because in that case it would be expected to diminish exponentially from the source, that is, with a very sharp spatial decay. Moreover, spreading depolarizations riding on a shallow negative ultraslow potential are also observed when cell death develops in the absence of a typical ischemic core region and, thus, in the absence of a classical terminal depolarization. This has been demonstrated in several models to lead to sustained massive depolarization, as during the tissue bath with externally applied high potassium solutions or with blockers of astrocytic metabolism (Herreras and Somjen 1993; Somjen 2001) or in the brain topical endothelin-1 (ET-1) model in rats (Fig. 7c) (Oliveira-Ferreira and others 2010). Clear evidence of negative ultraslow potentials has also been found in patients examined with subdural electrodes for electrocorticography. They were recorded in patients with aneurysmal subarachnoid hemorrhage in whom serial neuroimaging scans revealed that new delayed ischemic infarcts had developed during the monitoring period (Drenckhahn and others 2012).

The variety of in vivo models in which metabolic challenge to a large brain region is accompanied by a negative ultraslow potential points to a very basic 


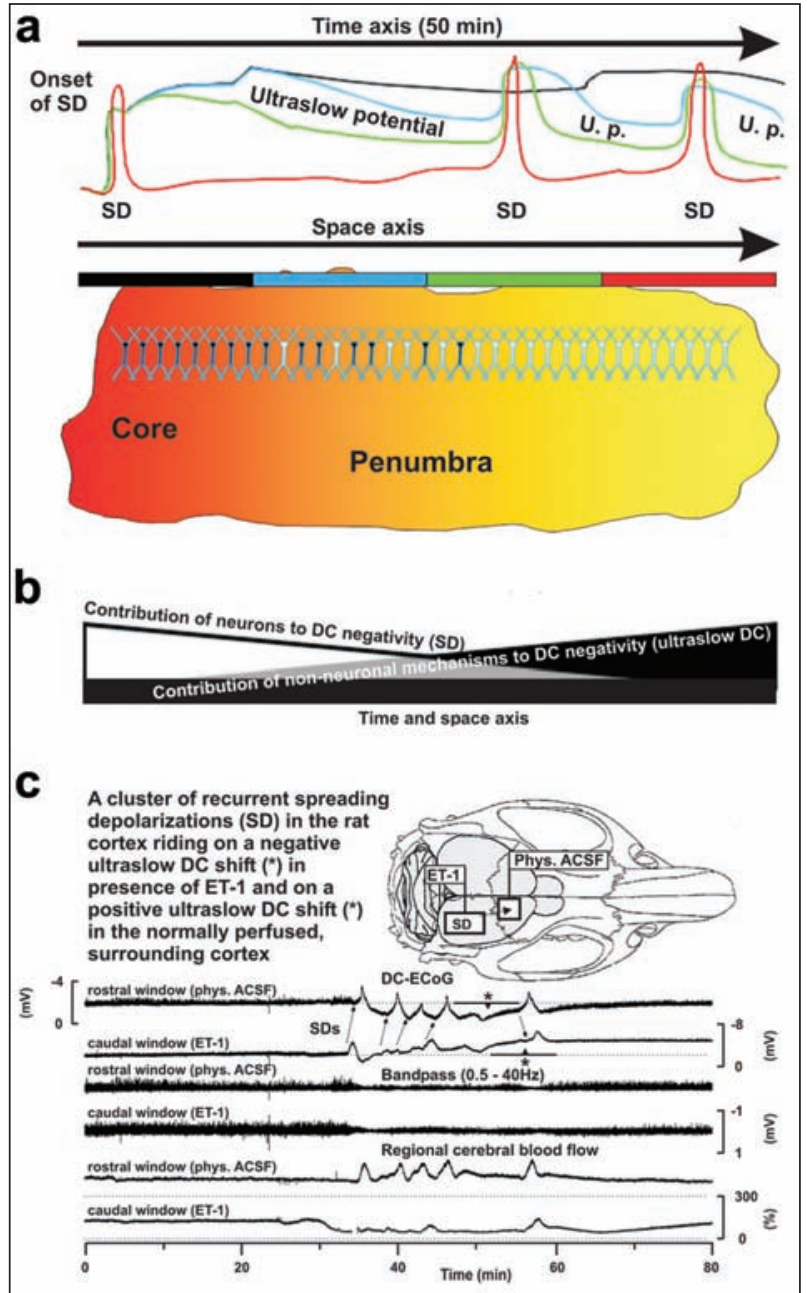

Figure 7. The different types of negative DC potentials are spatial and temporal landmarks for the energy status of neurons. (a) In severe focal ischemia after middle cerebral artery occlusion, for example, the initial spreading depolarization (SD) component starts in the center of the malperfused zone (black circle and recording trace) from where it spreads to the penumbra (blue and green circles and traces) and to surrounding cortex in which perfusion is increasingly normal (red circle and trace). The intracortical amplitude of this initial spreading depolarization component is typically somewhat smaller in the center of the malperfused cortex and increases toward the penumbra. In contrast, the ultraslow negative DC shift has maximum amplitude at the center of the malperfused cortex and decreases toward the penumbra.A double gradient of negative potentials is thus established between the ischemic center and the normally perfused cortex. The former becomes the necrotic core that expands in stepwise fashion into the penumbra following recurrent spreading depolarizations. This progression is paralleled by the ultraslow negativity progressively encroaching on farther zones, which implicates that the ultraslow negative DC shift captures the local evolution of the cell death in a single recording point. Time and space axes point along the propagation direction of the spreading depolarizations. (b) After the first spreading depolarization has established the necrotic core, the double gradient between the spreading depolarization component and the ultraslow potential is related to the neuron status and the neuronal and nonneuronal origin of the DC potentials. In healthy normoxic areas all neurons may contribute to the spreading depolarization negativity and their intact energy supply will let them recover quickly. In the penumbra region, the spreading depolarization negativity becomes smaller because there are fewer neurons alive the closer to the necrotic core. Those still alive enter in spreading depolarization but are energetically compromised and take longer to recover; hence, spreading depolarization waves last longer. Many neurons will not recover and add to the necrotic core. Thus, the next spreading depolarization wave will be smaller at that location. In the core, energy is exhausted and all cells including neurons have depolarized irreversibly; hence, they cannot promote the generation of transmembrane currents or field potentials. The generators of the ultraslow negativity are unknown but probably of non-neuronal origin, which might include both astrocytic and non-cellular mechanisms. (c) In milder ischemia, a cluster of recurrent spreading depolarizations (arrows) can also ride on a shallow negative ultraslow potential although a typical core region with terminal depolarization is lacking. An experiment in a rat with two cranial windows is given. At the caudal window, cortex was exposed to the vasoconstrictor ET-I whereas the rostral window was placed over naïve cortex. Traces I and 2 give the subdural DC-electrocorticographic (ECoG) potentials. Note the shallow negative DC shift (marked by *) on which spreading depolarizations are riding at the caudal window in contrast to the shallow positivity $(*)$ observed between the spreading depolarizations at the rostral window. Traces 3 and 4 demonstrate marked persistent depression of the high-frequency (HF)-ECoG activity, which is somewhat more pronounced in the ET-I exposed cortex. The lowest traces give the regional cerebral blood flow ( $\mathrm{rCBF}$ ). Note the significant decline of baseline rCBF before the cluster onset in the caudal window whereas it remained unchanged in the rostral window. ACSF, artificial cerebrospinal fluid. Modified with permission from Oliveira-Ferreira and others (2010).

physical mechanism. Lack of oxygen and/or glucose should be considered as trigger factors, but none is essential, as those potentials also develop in normoxic/ normoglycemic tissues. The area in the negative envelope is typically inundated with potassium and protons. At least the former has been considered to promote sustained negative potentials during epileptogenesis by current flow driven by inhomogeneous depolarization across the glial syncytium (Dietzel and others 1989). These are however small amplitude potentials and may be characteristic of ictal epileptic activity $/ G_{\text {mid }}$. Alternatively, non-cellular mechanisms may contribute to the genesis of ultraslow potentials (Fig. 7b). The affected regions have different ion concentrations compared with healthy tissue as a consequence of neuron membrane rupture and massive ion spillage. Passive ion diffusion across the extracellular space may establish sustained potential differences. 


\section{Clusters of Repeated Spreading Depolarizations}

The spatial and temporal recovery of the ion gradients after prolonged depolarizations under pathophysiological conditions as compared with the shorter depolarizations under physiological conditions has been studied in different models in vitro and in vivo (Dijkhuizen and others 1999; Herreras and Somjen 1993; Hossmann 1994; Oliveira-Ferreira and others 2010; Shin and others 2006; Windmuller and others 2005). Notably, cell death may ensue after mildly prolonged depolarizations in a delayed fashion although the ion gradients recovered transiently. For example, this was shown in a model in rats in which topical application of the potent vasoconstrictor ET-1 to the brain induced mild ischemia (Dreier and others 2002; Kleeberg and others 2004). The main difference between the ET-1 exposed ischemic cortex and the normally perfused surrounding cortex was that a cluster of repeated spreading depolarizations rode on a shallow negative ultraslow potential in the ischemic cortex (current sink) and on a shallow positive ultraslow potential in the normally perfused, surrounding cortex (current source) (Fig. 7c) (Oliveira-Ferreira and others 2010). The large negative DC shifts of the spreading depolarization waves were slightly longer in the ischemic zone; the neuronal activity was nevertheless persistently depressed between the recurrent spreading depolarization waves in both the ischemic and the normally perfused surrounding cortex (Fig. 7c).

Selective disruption of the aerobic metabolism in astrocytes by aconitase blockers is another model in which an ultraslow negative potential develops gradually in spatial and temporal concordance with a cluster of repeated spreading depolarizations and neuron death (Largo and others 1996). In this model, a cluster of recurrent spreading depolarizations suddenly occurred after a few hours lag. Although more delayed, the pattern of this cluster was reminiscent of that found in response to mild ischemia, but in the case of aconitase blockers the cluster occurred in normoxic and normoglycemic tissues. The cluster was accompanied by the loss of potassium buffering capability and $\mathrm{pH}$ control of the tissue, functions normally attributed to astrocytes (Benarroch 2005). Breakdown of the fine-tuned neurometabolic concert between astrocytes and neurons seems thus sufficient to produce a cascade of events remarkably similar to that in ischemia and with equally disastrous outcome. These findings correspond with the notion that the disturbance of glial function contributes to neuronal death in ischemia although astrocytes themselves are more resistant to ischemia than neurons thanks to their higher anaerobic capabilities.

Another interesting observation has been that clusters of spreading depolarizations in normoxic and normoglycemic tissue can trigger severe vasoconstriction and ischemia under specific conditions. This was discovered in an in vivo model in rats in which artificial cerebrospinal fluid of composition designed to replicate the conditions that follow aneurysmal subarachnoid hemorrhage was applied to the brain (Dreier and others 1998). In the presence of this abnormal cerebrospinal fluid, the resistance vessels responded to spreading depolarization with tone alterations causing ischemia (inverse hemodynamic response, or spreading ischemia) instead of the physiological hyperemic response. Parallel measurements of the DC potential, ion changes, and regional cerebral blood flow revealed that, in this particular condition, (a) the DC negativity and ion changes started earlier and lasted longer than the ischemic flow change; (b) the DC negativity, ion changes, and ischemic flow change spread in the brain; (c) the durations of DC negativity and ion changes were much prolonged compared with spreading depolarizations to which a physiological hyperemic flow response was coupled; and (d) the durations of DC negativity, and ischemic flow alterations correlated linearly (Windmuller and others 2005). The pathophysiological basis of such spreading ischemia is a vicious circle: (a) the neuronal depolarization causes the release of vasoconstrictors, which induce a severe decrease in blood flow; (b) the ischemia prolongs the neuronal depolarization because the recovery from the depolarization requires energy; (c) the continued neuronal depolarization causes the continued release of vasoconstrictors (Dreier 2011). In an analogous fashion, spreading ischemia was found in patients with aneurysmal subarachnoid hemorrhage (Dreier and others 2009). Similar to the experimental condition, (a) the DC negativity started before and outlasted the fall in cerebral blood flow, (b) the DC negativity and decline in blood flow spread in the tissue, (c) the spontaneous activity only recovered after the recovery from the DC negativity, and (d) linear correlations were observed between the durations of DC negativity, spreading ischemia, and spreading depolarization-induced depression of spontaneous activity.

These examples illustrate the complexity in clusters of repeated spreading depolarizations as a dynamic, nonlinear phenomenon of brain pathology, which displays significant variations in etiological factors, electrophysiological details, hemodynamic responses, and pharmacology (Dreier 2011). More experimental and clinical work is therefore needed to arrive at a more precise definition when and where in the tissue such clusters indicate significant damage to the brain. Why this is important is explained in the following.

\section{Conclusion}

A favorite approach to inform treatment decisions in medicine is to determine summary measures of an individual, also called biomarkers, which indicate injury to a 
specific organ. Such relatively simple but powerful summary measures exist for practically every organ of the body apart from the brain. The brain poses particular challenges because time from onset of an insult to damage is very short, brain structure and physiology are more complex, and the brain is less accessible to point-of-care diagnostic procedures and interventions than other organs because it is hidden behind skull and meninges.

Here we explain a concept, based on experimental data (Hansen and Zeuthen 1981; Kager and others 2002; Kraig and Nicholson 1978; Vorisek and Sykova 1997; Windmuller and others 2005) and on classical thermodynamic calculations, that spreading depolarization represents the Gibbs free energy state of the living brain closest to death. In addition to empirical arguments, this nature underlying spreading depolarization provides the theoretical justification for performing further empirical studies in the laboratory and clinic that may eventually establish routine neuromonitoring of spreading depolarizations as a real-time tool for intensive care medicine to detect acutely developing brain injury in sedated patients in whom clinical assessment is limited (Drenckhahn and others 2012). These electrophysiological tools and other tools for the assessment of energy metabolism including imaging modalities, sensors for oxygen or regional cerebral blood flow, and microdialysis are of course not mutually exclusive; indeed, they may actually be complementary.

Moreover, our theoretical concept supports the notion emerging from empirical data (Dohmen and others 2008; Dreier and others 2006; Fabricius and others 2006; Hartings and others 2011a) that spreading depolarizations may be an interesting mechanistic endpoint in clinical studies. Furthermore, a theoretical concept may help the discussion and exchange between the basic sciences and medical disciplines in this research field. Their collaborative effort will be necessary to answer the important questions. For example, whether it is indicated and how it is possible to stop spreading depolarization effectively in patients. This will have to be weighed in clinical and experimental studies against the potential interference of such treatment with processes of plasticity and repair that, in peri-ischemic tissue, are possibly promoted by spreading depolarization (Berger and others 2008; Dreier 2011; Lo 2008; Matsushima and others 1996; Yanamoto and others 2005).

For now, we have chosen two simple geometrical models to illustrate a basic thermodynamic concept of ictal epileptic activity and spreading depolarization because the available experimental data are limited. These models could be improved as further discussed in the Supplemental Material. However, as an initial step, our simple modeling exercise prognosticated in fairly good approximation the rise in tissue temperature and the release of heat during spreading depolarization that was experimentally measured in the retina (Tasaki and Byrne 1991). Within the limitations mentioned in this article, this interesting experimental observation seems to support the thermodynamic principles described here, which suggest an outstanding role of disturbances in ion homeostasis for brain pathology and justify further efforts to develop applications of this knowledge for the clinic.

\section{Acknowledgments}

We would like to thank Dr. George G. Somjen for reading the article and helpful discussions.

\section{Declaration of Conflicting Interests}

The author(s) declared no potential conflicts of interest with respect to the research, authorship, and/or publication of this article.

\section{Funding}

The author(s) disclosed receipt of the following financial support for the research, authorship, and/or publication of this article: Supported by grants of the Deutsche Forschungsgemeinschaft (DFG DR 323/3-1, 323/5-1, 323/6-1), the Bundesministerium für Bildung und Forschung (Center for Stroke Research Berlin, 01 EO 0801, Bernstein Center for Computational Neuroscience Berlin 01GQ1001C B2 and Era-Net Neuron SDSVD) to Dr. Dreier and the National Institutes of Health, NS057113 to Dr. Kirov.

\section{References}

Alle H, Roth A, Geiger JR. 2009. Energy-efficient action potentials in hippocampal mossy fibers. Science 325(5946):1405-8.

Astrup J, Symon L, Branston NM, Lassen NA. 1977. Cortical evoked potential and extracellular $\mathrm{K}^{+}$and $\mathrm{H}^{+}$at critical levels of brain ischemia. Stroke 8(1):51-7.

Avoli M, Drapeau C, Louvel J, Pumain R, Olivier A, Villemure JG. 1991. Epileptiform activity induced by low extracellular magnesium in the human cortex maintained in vitro. Ann Neurol 30(4):589-96.

Attwell D, Laughlin SB. 2001. An energy budget for signaling in the grey matter of the brain. J Cereb Blood Flow Metab 21(10):1133-45.

Benarroch EE. 2005. Neuron-astrocyte interactions: partnership for normal function and disease in the central nervous system. Mayo Clin Proc 80(10):1326-38.

Berger M, Speckmann EJ, Pape HC, Gorji A. 2008. Spreading depression enhances human neocortical excitability in vitro. Cephalalgia 28(5):558-62.

Branston NM, Strong AJ, Symon L. 1977. Extracellular potassium activity, evoked potential and tissue blood flow. Relationships during progressive ischaemia in baboon cerebral cortex. J Neurol Sci 32(3):305-21.

Bures J, Buresova O. 1957. Die anoxische Terminaldepolarisation als Indikator der Vulnerabilität der Großhirnrinde bei Anoxie und Ischämie. Pflügers Arch 264(4):325-34. 
Canals S, Makarova I, Lopez-Aguado L, Largo C, Ibarz JM, Herreras O. 2005. Longitudinal depolarization gradients along the somatodendritic axis of CA1 pyramidal cells: a novel feature of spreading depression. J Neurophysiol 94(2):943-51.

Charriaut-Marlangue C, Margaill I, Represa A, Popovici T, Plotkine M, Ben-Ari Y. 1996. Apoptosis and necrosis after reversible focal ischemia: an in situ DNA fragmentation analysis. J Cereb Blood Flow Metab 16(2):186-94.

Dalkara T, Nozari A, Moskowitz MA. 2010. Migraine aura pathophysiology: the role of blood vessels and microembolisation. Lancet Neurol 9(3):309-17.

Dietzel I, Heinemann U, Lux HD. 1989. Relations between slow extracellular potential changes, glial potassium buffering, and electrolyte and cellular volume changes during neuronal hyperactivity in cat brain. Glia 2(1):25-44.

Dijkhuizen RM, Beekwilder JP, van der Worp HB, Berkelbach van der Sprenkel JW, Tulleken KA, Nicolay K. 1999. Correlation between tissue depolarizations and damage in focal ischemic rat brain. Brain Res 840(1-2):194-205.

Dohmen C, Sakowitz OW, Fabricius M, Bosche B, Reithmeier T, Ernestus RI, and others. 2008. Spreading depolarizations occur in human ischemic stroke with high incidence. Ann Neurol 63(6):720-8.

Dreier JP. 2011. The role of spreading depression, spreading depolarization and spreading ischemia in neurological disease. Nat Med 17(4):439-47.

Dreier JP, Heinemann U. 1991. Regional and time dependent variations of low $\mathrm{Mg}^{2+}$ induced epileptiform activity in rat temporal cortex slices. Exp Brain Res 87(3):581-96.

Dreier JP, Kleeberg J, Petzold G, Priller J, Windmuller O, Orzechowski HD, and others. 2002. Endothelin-1 potently induces Leao's cortical spreading depression in vivo in the rat: a model for an endothelial trigger of migrainous aura? Brain 125(Pt 1):102-12.

Dreier JP, Korner K, Ebert N, Gorner A, Rubin I, Back T, and others. 1998. Nitric oxide scavenging by hemoglobin or nitric oxide synthase inhibition by N-nitro-L-arginine induces cortical spreading ischemia when $\mathrm{K}^{+}$is increased in the subarachnoid space. J Cereb Blood Flow Metab 18(9):978-90.

Dreier JP, Major S, Manning A, Woitzik J, Drenckhahn C, Steinbrink J, and others. 2009. Cortical spreading ischaemia is a novel process involved in ischaemic damage in patients with aneurysmal subarachnoid haemorrhage. Brain 132(Pt 7):1866-81.

Dreier JP, Major S, Pannek HW, Woitzik J, Scheel M, Wiesenthal D, and others. 2012. Spreading convulsions, spreading depolarization and epileptogenesis in human cerebral cortex. Brain 135(Pt 1):259-75.

Dreier JP, Woitzik J, Fabricius M, Bhatia R, Major S, Drenckhahn C, and others. 2006. Delayed ischaemic neurological deficits after subarachnoid haemorrhage are associated with clusters of spreading depolarizations. Brain 129(Pt 12):3224-37.
Drenckhahn C, Winkler MKL, Major S, Scheel M, Kang EJ, Pinczolits A, and others. 2012. Correlates of spreading depolarizations in human scalp electroencephalography. Brain 135(Pt 3):853-68.

Eikermann-Haerter K, Hyun Lee J, Yuzawa I, Liu CH, Zhou Z, Kyoung Shin H, and others. 2012. Migraine mutations increase stroke vulnerability by facilitating ischemic depolarizations. Circulation 125(2):335-45.

Fabricius M, Fuhr S, Bhatia R, Boutelle M, Hashemi P, Strong AJ, and others. 2006. Cortical spreading depression and periinfarct depolarization in acutely injured human cerebral cortex. Brain 129(Pt 3):778-90.

Fabricius M, Fuhr S, Willumsen L, Dreier JP, Bhatia R, Boutelle MG, and others. 2008. Association of seizures with cortical spreading depression and peri-infarct depolarisations in the acutely injured human brain. Clin Neurophysiol 119(9):1973-84.

Gerich FJ, Hepp S, Probst I, Muller M. 2006. Mitochondrial inhibition prior to oxygen-withdrawal facilitates the occurrence of hypoxia-induced spreading depression in rat hippocampal slices. J Neurophysiology 96(1):492-504.

Gorji A, Scheller D, Straub H, Tegtmeier F, Kohling R, Hohling JM, and others. 2001. Spreading depression in human neocortical slices. Brain Res 906(1-2):74-83.

Grafstein B. 1956. Mechanism of spreading cortical depression. J Neurophysiol 19(2):154-71.

Hadjikhani N, Sanchez Del Rio M, Wu O, Schwartz D, Bakker D, Fischl B, and others. 2001. Mechanisms of migraine aura revealed by functional MRI in human visual cortex. Proc Natl Acad Sci U S A 98(8):4687-92.

Hansen AJ, Zeuthen T. 1981. Extracellular ion concentrations during spreading depression and ischemia in the rat brain cortex. Acta Physiol Scand 113(4):437-45.

Hartings JA, BullockMR, Okonkwo DO, Murray LS, Murray GD, Fabricius M, and others. 2011a. Spreading depolarisations and outcome after traumatic brain injury: a prospective observational study. Lancet Neurol 10(12):1058-64.

Hartings JA, Watanabe T, Bullock MR, Okonkwo DO, Fabricius M, and others. Woitzik J, and others. 2011b. Spreading depolarizations have prolonged direct current shifts and are associated with poor outcome in brain trauma. Brain 134 1529-40.

Heinemann U, Lux HD. 1977. Ceiling of stimulus induced rises in extracellular potassium concentration in the cerebral cortex of cat. Brain Res 120(2):231-49.

Herrera FC. 1992. The partial double Donnan - a simple alternative interpretation of the transmembrane ionic distribution. Comp Biochem Physiol 103A:29-34.

Herreras O, Largo C, Ibarz JM, Somjen GG, Martin del Rio R. 1994. Role of neuronal synchronizing mechanisms in the propagation of spreading depression in the in vivo hippocampus. J Neurosci 14(11 Pt 2):7087-98.

Herreras O, Somjen GG. 1993. Analysis of potential shifts associated with recurrent spreading depression and prolonged 
unstable spreading depression induced by microdialysis of elevated $\mathrm{K}^{+}$in hippocampus of anesthetized rats. Brain Res 610(2):283-94.

Hossmann KA. 1994. Viability thresholds and the penumbra of focal ischemia. Ann Neurol 36(4):557-65.

Kager H, Wadman WJ, Somjen GG. 2002. Conditions for the triggering of spreading depression studied with computer simulations. J Neurophysiol 88(5):2700-12.

Kleeberg J, Petzold GC, Major S, Dirnagl U, Dreier JP. 2004. ET-1 induces cortical spreading depression via activation of the ETA receptor/phospholipase C pathway in vivo. Am J Physiol Heart Circ Physiol 286(4):H1339-46.

Kraig RP, Nicholson C. 1978. Extracellular ionic variations during spreading depression. Neuroscience 3(11):1045-59.

Kurth T, Chabriat H, Bousser MG. 2012. Migraine and stroke: a complex association with clinical implications. Lancet Neurol 11(1):92-100.

LaManna JC, Rosenthal M. 1975. Effect of ouabain and phenobarbital on oxidative metabolic activity associated with spreading cortical depression in cats. Brain Res 88(1): 145-9.

Largo C, Cuevas P, Somjen GG, Martin del Rio R, Herreras O. 1996. The effect of depressing glial function in rat brain in situ on ion homeostasis, synaptic transmission, and neuron survival. J Neurosci 16(3):1219-29.

Lauritzen M. 1994. Pathophysiology of the migraine aura. The spreading depression theory. Brain 117(Pt 1):199-210.

Leaf A. 1956. On the mechanism of fluid exchange of tissues in vitro. Biochem J 62(2):241-8.

Leão AAP. 1944a. Pial circulation and spreading depression of activity in the cerebral cortex. J Neurophysiol 7:391-6.

Leão AAP. 1944b. Spreading depression of activity in the cerebral cortex. J Neurophysiol 7:359-90.

Leão AAP. 1947. Further observations on the spreading depression of activity in the cerebral cortex. J Neurophysiol 10:409-14.

Leão AAP, Morison RS. 1945. Propagation of spreading cortical depression. J Neurophysiol 8:33-45.

Lo EH. 2008. A new penumbra: transitioning from injury into repair after stroke. Nat Med 14(5):497-500.

Makarova J, Makarov VA, Herreras O. 2010. Generation of sustained field potentials by gradients of polarization within single neurons: a macroscopic model of spreading depression. J Neurophysiol 103(5):2446-57.

Martins-Ferreira H, De Oliveira Castro G, Struchiner CJ, Rodrigues PS. 1974. Circling spreading depression in isolated chick retina. J Neurophysiol 37(4):773-84.

MaslarovaA,Alam M, Reiffurth C, LapiloverE, GorjiA, Dreier JP. 2011. Chronically epileptic human and rat neocortex display a similar resistance against spreading depolarization in vitro. Stroke 42(10):2917-22.

Matsushima K, Hogan MJ, Hakim AM. 1996. Cortical spreading depression protects against subsequent focal cerebral ischemia in rats. J Cereb Blood Flow Metab 16(2):221-6.

Mies G, Iijima T, Hossmann KA. 1993. Correlation between peri-infarct DC shifts and ischaemic neuronal damage in rat. Neuroreport 4(6):709-11.

Mies G, Paschen W. 1984. Regional changes of blood flow, glucose, and ATP content determined on brain sections during a single passage of spreading depression in rat brain cortex. Exp Neurol 84(2):249-58.

Mody I, Lambert JD, Heinemann U. 1987. Low extracellular magnesium induces epileptiform activity and spreading depression in rat hippocampal slices. J Neurophysiol 57(3):869-88.

Muller M, Somjen GG. 2000. $\mathrm{Na}(+)$ and $\mathrm{K}(+)$ concentrations, extra- and intracellular voltages, and the effect of TTX in hypoxic rat hippocampal slices. J Neurophysiol 83(2):735-45.

Nakamura H, Strong AJ, Dohmen C, Sakowitz OW, Vollmar S, Sue M, and others. 2010. Spreading depolarizations cycle around and enlarge focal ischaemic brain lesions. Brain 133(Pt 7):1994-2006.

Nedergaard M, Astrup J. 1986. Infarct rim: effect of hyperglycemia on direct current potential and [14C]2-deoxyglucose phosphorylation. J Cereb Blood Flow Metab 6(5):607-15.

Ohta K, Graf R, Rosner G, Heiss WD. 2001. Calcium ion transients in peri-infarct depolarizations may deteriorate ion homeostasis and expand infarction in focal cerebral ischemia in cats. Stroke 32(2):535-43.

Olesen J, Larsen B, Lauritzen M. 1981. Focal hyperemia followed by spreading oligemia and impaired activation of rCBF in classic migraine. Ann Neurol 9(4):344-52.

Oliveira-Ferreira AI, Milakara D, Alam M, Jorks D, Major S, Hartings JA, and others. 2010. Experimental and preliminary clinical evidence of an ischemic zone with prolonged negative DC shifts surrounded by a normally perfused tissue belt with persistent electrocorticographic depression. J Cereb Blood Flow Metab 30(8):1504-19.

Oliveira-Ferreira AI, Winkler MKL, Reiffurth C, Milakara D, Woitzik J, Dreier JP. 2012. Spreading depolarization, a pathophysiological mechanism of stroke and migraine aura. Future Neurol 7:45-64.

Risher WC, Andrew RD, Kirov SA. 2009. Real-time passive volume responses of astrocytes to acute osmotic and ischemic stress in cortical slices and in vivo revealed by twophoton microscopy. Glia 57(2):207-21.

Risher WC, Ard D, Yuan J, Kirov SA. 2010. Recurrent spontaneous spreading depolarizations facilitate acute dendritic injury in the ischemic penumbra. J Neurosci 30(29):9859-68.

Selman WR, Lust WD, Pundik S, Zhou Y, Ratcheson RA. 2004. Compromised metabolic recovery following spontaneous spreading depression in the penumbra. Brain Res 999(2):167-74.

Shapiro BE. 2001. Osmotic forces and gap junctions in spreading depression: a computational model. J Comput Neurosci 10(1):99-120. 
Shin HK, Dunn AK, Jones PB, Boas DA, Moskowitz MA, Ayata C. 2006. Vasoconstrictive neurovascular coupling during focal ischemic depolarizations. J Cereb Blood Flow Metab 26(8):1018-30.

Somjen GG. 2001. Mechanisms of spreading depression and hypoxic spreading depression-like depolarization. Physiol Rev 81(3):1065-96.

Somjen GG. 2004. Ions in the brain. Oxford: Oxford University Press.

Strong AJ, Fabricius M, Boutelle MG, Hibbins SJ, Hopwood SE, Jones R, and others. 2002. Spreading and synchronous depressions of cortical activity in acutely injured human brain. Stroke 33(12):2738-43.

Takano T, Tian GF, Peng W, Lou N, Lovatt D, Hansen AJ, and others. 2007. Cortical spreading depression causes and coincides with tissue hypoxia. Nat Neurosci 10(6):754-62.

Tanaka E, Yamamoto S, Kudo Y, Mihara S, Higashi H. 1997. Mechanisms underlying the rapid depolarization produced by deprivation of oxygen and glucose in rat hippocampal CA1 neurons in vitro. J Neurophysiol 78(2):891-902.

Tasaki I, Byrne PM. 1991. Demonstration of heat production associated with spreading depression in the amphibian retina. Biochem Biophys Res Commun 174(1):293-7.

Tidow H, Aperia A, Nissen P. 2010. How are ion pumps and agrin signaling integrated? Trends Biochem Sci 35(12): 653-9.
Umegaki M, Sanada Y, Waerzeggers Y, Rosner G, Yoshimine T, Heiss WD, and others. 2005. Peri-infarct depolarizations reveal penumbra-like conditions in striatum. J Neurosci 25(6):1387-94.

Van Harreveld A. 1959. Compounds in brain extracts causing spreading depression of cerebral cortical activity and contraction of crustacean muscle. J Neurochem 3:300-315.

Van Harreveld A, Crowell J, Malhotra SK. 1965. A study of extracellular space in central nervous tissue by freezesubstitution. J Cell Biol 25:117-37.

Vorisek I, Sykova E. 1997. Ischemia-induced changes in the extracellular space diffusion parameters, $\mathrm{K}^{+}$, and $\mathrm{pH}$ in the developing rat cortex and corpus callosum. J Cereb Blood Flow Metab 17(2):191-203.

Windmuller O, Lindauer U, Foddis M, Einhaupl KM, Dirnagl U, Heinemann U, and others. 2005. Ion changes in spreading ischaemia induce rat middle cerebral artery constriction in the absence of NO. Brain 128(Pt 9):2042-51.

Yanamoto H, Miyamoto S, Tohnai N, Nagata I, Xue JH, Nakano Y, and others. 2005. Induced spreading depression activates persistent neurogenesis in the subventricular zone, generating cells with markers for divided and early committed neurons in the caudate putamen and cortex. Stroke 36(7):1544-50. 